\title{
Quaderni
}

QUADERN I Communication, technologies, pouvoir

67 | Automne 2008

Jeu vidéo et discours

\section{Après le Moment Constitutionnel : la régulation des mondes virtuels 2.0}

After the Constitutional Moment: Regulating Virtual Worlds 2.0

\section{Viktor Mayer-Schönberger}

Traducteur : Olivier Mauco

\section{OpenEdition}

Journals

Édition électronique

URL : http://journals.openedition.org/quaderni/221

DOI : 10.4000/quaderni.221

ISSN : 2105-2956

Éditeur

Les éditions de la Maison des sciences de l'Homme

Édition imprimée

Date de publication : 5 octobre 2008

Pagination : 75-84

\section{Référence électronique}

Viktor Mayer-Schönberger, « Après le Moment Constitutionnel : la régulation des mondes virtuels $2.0 »$, Quaderni [En ligne], 67 | Automne 2008, mis en ligne le 05 janvier 2012, consulté le 22 avril 2019. URL : http://journals.openedition.org/quaderni/221 ; DOI : 10.4000/quaderni.221 


\section{$D$ ossier}

\section{après le Moment Constitutionnel :}

la régulation des mondes virtuels 2.0

Partout dans le monde, plus de dix millions de personnes arpentent des mondes virtuels. Des centaines de millions d'euros sont générés par les fournisseurs de ces mondes, mais des milliards sont issus de la négociation des biens virtuels sur des marchés virtuels. Il ne s'agit pas seulement d'un Monopoly des temps modernes. Bien que ces transactions soient en monnaie virtuelle, ces devises sont entièrement convertibles en dollars US, ce qui permet facilement des flux d'entrées et de sorties de capitaux. Dégagés de tout système hiérarchique pyramidal, les utilisateurs participent à des opérations du marché bien réelles, comme par exemple sur e-bay, alimenté par la réalité de l'offre et de la demande de ces biens.

\section{Viktor \\ Mayer-Schönberger}

Harvard Kennedy School of government

Ainsi, au vu d'un tel nombre de participants et de transactions, les conflits sont voués à éclater. Et ils éclatent. Chaque monde virtuel comporte son lot d'histoires de malentendus, de fraude et de comportement criminel - tout comme le monde réel. Le but de cet article n'est pas tant de savoir si certains comportements dans les mondes virtuels devraient être ou ne pas être réglementés, ni par qui. D'autres ont développé cette question ${ }^{1}$, et les points de vue varient d'une juridiction à une autre, le point central de ces variations relevant essentiellement des conflits de compétence juridictionnelle, relatifs à l'émergence du commerce électronique.

Au lieu de cela, cet article entend dans un premier temps révéler le domaine des possibles et les contraintes relatives à la réglementation des mondes virtuels. Dans un second temps, cet article analyse en quoi le paysage concurrentiel des fournisseurs de mondes virtuels est en inte- 
des mondes virtuels soutenues par les décideurs politiques du monde réel.

\section{Les mondes émergents}

Les mondes virtuels sont un phénomène assez récent. Dès les années 1990, les ordinateurs bon marché rendent possible les jeux vidéo en trois dimensions. Les réseaux numériques mondiaux, en particulier l'Internet, nous ont permis de connecter des ordinateurs personnels (et à travers eux les utilisateurs) à des serveurs centraux, quelle que soit la distance géographique. Ces facteurs ont facilité la croissance de ce que l'on appelle les MMORPG - les jeux de rôles massivement multi-joueurs en ligne. Everquest (Sony Online Entertainment) et Lineage (NCSoft), notamment en Corée du Sud, sont les premiers mondes en ligne à devenir des blockbusters, mais se sont vus depuis dépassés par World of Warcraft (Vivendi, Blizzard Entertainment).

Ces mondes de synthèse, néanmoins, demeurent fondamentalement des jeux; ils offrent aux utilisateurs un telos unificateur, un objectif qu'ils doivent atteindre ou une tâche qu'ils ont à remplir. En outre, l'intégralité du monde que les utilisateurs connaissent est construite et gérée par le fournisseur du jeu. Les utilisateurs sont libres de les arpenter et parfois même de faire de petites modifications sur l'environnement, mais en général, ils se trouvent enserrés dans des espaces virtuels préfabriqués où une autorité centrale $-\mathrm{le}$ fournisseur du jeu - a soigneusement défini les possibilités d'interaction des utilisateurs avec leur environnement, en vertu du game play, des possibilités d'interaction, des règles et du game design.
À l'opposé, les mondes virtuels ne présentent pas de telos que les utilisateurs devraient poursuivre, et leur contenu n'est pas intégralement créé par le fournisseur. Au contraire, les utilisateurs sont invités à construire et à développer le (relativement stérile) monde virtuel qu'ils intègrent; le monde est construit et développé au fil du temps. Ces deux caractéristiques ont été empruntées aux univers en ligne basés sur du texte (MUD) du début des années 1990, dont les plus connus sont Lambda-MOO (1990) et MediaMOO (1993, MIT Media Lab). Or dans notre cas, les espaces virtuels sont en trois dimensions, graphiquement riches, pouvant imiter les lois de la physique, y compris la gravité, reproduire les jeux d'ombre et de lumière. De puissants outils logiciels permettent aux utilisateurs de d'y construire des objets en trois dimensions, et les langages scriptés de comportements de donner aux objets qu'ils ont créés des fonctions distinctes (et complexes) ${ }^{2}$.

Le choix d'offrir un monde virtuel créé par l'utilisateur, plutôt qu'un monde synthétique préfabriqué, a des répercussions économiques énormes pour les fournisseurs. Alors que les fournisseurs des MMO doivent employer des dizaines, voire des centaines de développeurs de contenu à plein temps, les fournisseurs de mondes virtuels peuvent concentrer leurs ressources sur la création d'un code-source qui structure le monde, tandis que les utilisateurs façonneront eux-mêmes le monde virtuel. De manière organisationnelle, les développeurs de monde virtuel ont externalisé le développement du contenu à leurs propres utilisateurs, qui peuvent même payer un abonnement mensuel pour contribuer à la création de contenu. Malgré la complexité des outils de construction, le temps et les efforts nécessaires pour créer des 
objets dans ces mondes virtuels, les utilisateurs n'ont de cesse de construire intensément. Second Life apparaît sans doute comme le monde virtuel le plus réussi à ce jour, contenant par exemple plusieurs centaines de millions d'objets créés par les utilisateurs ; et le nombre de codes écrits par les utilisateurs-créateurs d'objets dans Second Life dépasse de beaucoup la totalité des contributions pour Linux. En outre, la capacité de créer a donné lieu à la spécialisation de l'utilisateur et à la création de marchés vivants pour objets virtuels, des constructions et architectures virtuelles, des courtiers virtuels, et on voit même apparaître un marché de l'immobilier virtuel.

Sur le long terme, les mondes de synthèse, de par la création de contenu centralisée (et donc coûteux), auront beaucoup de difficultés à lutter avec le succès des mondes virtuels. Pour concurrencer la variété et la quantité de contenus créés pour Second Life, le fournisseur d'un monde de synthèse traditionnel devrait embaucher des milliers de designers et de codeurs, et ce pendant des années avant d'être en mesure de rattraper leur retard en termes de quantité et de variété proposées par des mondes virtuels comme $\mathrm{Se}$ cond Life. Bien entendu, la création de contenu centralisée a ses vertus. La création peut être étroitement contrôlée, et l'expérience de jeu peut être esthétique et agréable. Dans les mondes de synthèse, tous les objets, et la plupart des choses en fait, sont programmés pour fonctionner d'une certaine manière. En revanche, les mondes virtuels sont remplis de projets à moitié inachevés ou en cours de construction, voire de chantiers abandonnés. Pour l'utilisateur moyen, l'origine et l'utilisation de nombreux objets sont parfois difficiles à cerner, et même les objets peuvent avoir des comportements non désirés en raison d'erreurs de codage et d'erreurs de conception. Pour certains utilisateurs cette contrainte technique pourrait réduire l'attrait des mondes virtuels. Mais les mondes virtuels inachevés sont aussi en constante évolution, et offrent sans cesse de nouveaux domaines à explorer, et de nouvelles possibilités de contribution. Ils sont également beaucoup plus proches de notre monde physique, dans lequel nous rencontrons beaucoup de choses étranges, parce que nous ne parvenons pas à les comprendre ou parce qu'elles n'ont pas encore été terminées. Il en est de même pour l'absence d'un telos central et prédéterminé. Ces mondes virtuels ne parviennent pas à fournir un sens à la cohésion sociale, un but commun, mais en même temps ils représentent pour les utilisateurs un espace dans lequel façonner son propre telos, pour eux et/ou pour les autres.

De manière un peu simplifiée, les mondes synthétiques sont des sociétés planifiées, tandis que les mondes virtuels sont fondamentalement ouverts, divers et hétérogènes, en offrant des choix grâce au marché et aux opportunités. Nous pouvons alors saisir qu'avec le temps ces jardins d'hiver à la planification centralisée perdront contre le chaos fascinant de la diversité et de la créativité humaine décentralisée - comme toutes les planifications centralisées ont perdu contre la diversité du marché. Sur le long terme, donc, une grande partie de l'activité des utilisateurs pourrait migrer des mondes synthétiques vers les mondes virtuels, nous conduisant sans doute à une situation dans laquelle un certain nombre de mondes virtuels seront en concurrence les uns contre les autres pour capter les utilisateurs (ou dans la langue de la mondialisation, en concurrence pour le capital 
et la connaissance).

La concurrence des mondes virtuels et le Moment constitutionnel

Cette concurrence entre prestataires de mondes virtuels se fonde sur deux éléments distincts : chaque fournisseur tente d'attirer de nouveaux utilisateurs et en même temps essaie de conserver le plus grand nombre d'utilisateurs possible. Stratégiquement, les fournisseurs ont rendu leurs mondes faciles d'accès, et très difficiles à quitter. Les transferts de monde à monde sont coûteux et difficiles car, après tout, les migrations nécessitent pour les utilisateurs de passer à un nouveau système, un nouveau mode de fonctionnement. Deuxièmement, la migration impose d'abandonner derrière soi les liens sociaux et les amitiés virtuelles - sauf si un groupe décide de changer de monde (ce qui se produit plus souvent qu'on ne l'imagine). La monnaie virtuelle crée une troisième modalité de verrouillage, bien que la convertibilité des devises virtuelles ait éliminé en grande partie les coûts de commutation associés. Enfin, même si les fournisseurs permettent aux utilisateurs de créer leurs propres objets dans les mondes virtuels, les fournisseurs ont traditionnellement des droits patrimoniaux et de propriété intellectuelle sur tous les objets, y compris ceux créés par l'utilisateur. Cela constitue un moyen de blocage puissant, car la migration vers un autre monde sous-entend l'abandon de toutes ses créations virtuelles, et donc la nécessité de repartir à zéro. Ce d'autant plus pour les utilisateurs qui ont des finalités commerciales, qui seraient alors désemparés.

Une décision capitale a changé la donne il y a quelques années : un fournisseur de mondes virtuels, Linden Lab, a accepté de permettre aux utilisateurs de Second Life de conserver leurs droits de propriété intellectuelle ${ }^{3}$. Ce qui a première vue pourrait sembler une stratégie discutable, étant donné que cela réduit fortement les coûts de migration, s'est avéré une stratégie fascinante, car dès lors que les utilisateurs ont la capacité technique de construire et de conserver leurs droits de propriété intellectuelle, ils perçoivent le monde virtuel comme étant réellement le leur. Ce coup stratégique a généré des flux migratoires d'utilisateurs conséquents vers le monde virtuel de Linden Lab et a augmenté la création de contenu. Second Life est devenu l'un des mondes virtuels les plus peuplés, avec régulièrement plus de trente mille utilisateurs quotidiens connectés et plus d'un demi-million d'utilisateurs fréquents.

Les plus critiques ont accusé l'initiative Linden Lab de n'être qu'une annonce publicitaire. À quoi cela sert-il d'avoir des droits de propriété intellectuelle, suggèrent-ils, quand l'utilisateur n'a pas la possibilité d'extraire des biens virtuels de Second Life et de les insérer dans un autre monde virtuel? L'importation et l'exportation de biens virtuels dans les mondes virtuels est en effet une question épineuse. Sans une technologie standardisée pour permettre l'exportation, les coûts réels pour les utilisateurs restent élevés. En outre, l'analyse économique impose que les fournisseurs de mondes virtuels assurent le plus grand nombre possible d'importateurs, mais en aucun cas n'encouragent à prendre des mesures pour faciliter l'exportation. En d'autres termes, les prestataires ont une forte incitation à s'emparer d'autres fournisseurs, ce afin de permettre 
l'exportation, mais également une forte incitation à interdire l'exportation de biens virtuels dans leur propre monde.

La dynamique entre les fournisseurs de mondes virtuels - comme l'ont suggéré les commentateurs - sera compétitive et agressive, sans avoir besoin d'une coopération inter-mondes. Dans mon article, «Napster's Second Life», j'ai discuté ce point ${ }^{4}$, le considérant comme fondé sur des conjectures hasardeuses. J'ai suggéré que, dans une certaine mesure, selon la logique du dilemme du prisonnier, la coopération entre les mondes virtuels et les fournisseurs sur la question des migrations serait un moyen pour assurer aux fournisseurs qui coopèrent une certaine suprématie.

Fait intéressant, c'est ce qui s'est réellement passé dans la pratique. À l'automne 2007, les principaux fournisseurs de mondes virtuels et des tiers importants se sont réunis et ont convenu d'élaborer des normes communes pour permettre la persistance et l'interopérabilité entre les mondes virtuels. Évidemment, il ne s'agit que d'une première étape, mais elle est importante, et inspire un important mouvement de coordination au sein de cette autrement grande dynamique concurrentielle des mondes virtuels ${ }^{5}$.

Dans l'ensemble, l'octroi de droits de propriété intellectuelle, l'établissement de la persistance et l'interopérabilité mènent à ce que je voudrais qualifier de «moment constitutionnel», un moment dans l'évolution des mondes virtuels où grâce à l'octroi de droits sur des biens virtuels, l'équilibre des pouvoirs entre le fournisseur et la communauté des utilisateurs a basculé en faveur des utilisateurs - non pas individuellement, bien sûr, mais collectivement. Je pense qu'il s'agit d'un moment constitutionnel, c'est-à-dire un moment après quoi les fournisseurs ont perdu une part importante de leur contrôle sur leur monde virtuel, et ce pour deux raisons. Tout d'abord, parce qu'elle ancre les droits des utilisateurs sur les biens virtuels sur les concepts de la propriété intellectuelle du monde réel. Deuxièmement ne pas garantir les droits sur les biens virtuels, en particulier par un fournisseur seul, serait très délicat, car cela signifierait exclure une base d'utilisateurs, et ce dans un contexte de marché concurrentiel.

\section{Les dynamiques de réglementation et l'équilibre temporaire}

Alors que les fournisseurs de mondes synthétiques sont en concurrence les uns contre les autres sur la nature de leur telos (l'objet offert aux utilisateurs), sur le contenu (la quantité et la qualité de leurs mondes), les fournisseurs de mondes virtuels ne peuvent pas entrer en compétition avec eux, et ne risqueraient pas de peser suffisamment. Pour eux, le contenu est largement fonction de la taille de leur population d'utilisateurs, bien que l'on puisse imaginer un cas extrême où une petite population très ciblée d'utilisateurs spécialisés pourrait être en mesure de concurrencer avec succès de grands mondes virtuels dont la communauté d'utilisateurs est relativement inactive. Cela limite les possibilités d'avantages concurrentiels.

En plus du contenu, les prestataires peuvent également se concurrencer sur le prix (abonnement mensuel, frais d'utilisation, etc), bien que le prix soit rarement un critère de distinction 
pour un tel service ; le danger de banalisation est trop fort lorsque le prix devient le centre de la compétition.

En conséquence, il est probable que la troisième et dernière dimension compétitive gagne de l'importance. Cette dimension se concentre sur les modalités de gestion du monde virtuel par le fournisseur, c'est-à-dire comment en tant que prestataire il peut offrir un monde conforme aux attentes et aux préférences d'une population d'utilisateurs ciblée.

Dans la mesure où cette évolution nous mène vers cette troisième dimension compétitive, nous allons voir que le paysage des mondes virtuels dynamiques est similaire à celui du monde réel, avec ses agents clefs en concurrence pour les ressources - les capitaux, les personnes et la connaissance. Dans le monde réel, la mobilité des ressources des États nécessite et permet une concurrence intensive. Plus ces ressources seront mobiles, plus la concurrence inter-étatique s'emballera.

Comme les ressources virtuelles deviennent mobiles, les mondes virtuels devraient nous fournir un terrain d'application intéressant pour les théories de la dynamique de la réglementation, mises en avant par les chercheurs en économie politique internationale ${ }^{6}$. Déjà, certains voient les mondes virtuels engagés dans une concurrence en matière réglementaire, course où les fournisseurs les moins restrictifs devraient emporter le plus grand nombre d'utilisateurs. Toutefois, avant d'arriver à de telles conclusions, nous devons comprendre non seulement les limites des théories de l'interaction de réglementation - les prédictions d'une « course effrénée » se révèlent être forte- ment surestimées ${ }^{7}$ - mais surtout les contraintes des mondes virtuels qui limitent la comparaison avec la dynamique du monde réel. Par exemple, étant donnée la faiblesse des coûts d'entrée pour un fournisseur de monde virtuel (et peut-être à moyen terme le faible coût de la migration entre ces mondes), la convergence de tous les utilisateurs sur un monde particulier (par exemple le moins réglementé, le mieux réglementé, ou dont la réglementation est la plus proche de la réalité des utilisateurs) est moins probable que le développement d'un large éventail de mondes virtuels ciblant les préférences des profils utilisateurs. Plutôt que d'atteindre le graal, un monde parfaitement régulé, il apparaît davantage probable que nous nous retrouvions dans la situation de l'équilibre temporaire de Tiebout ${ }^{8}$ : un océan de mondes-niches couvrant tout le spectre, de la permissive Las Vegas virtuelle à l'efficace Singapour virtuelle, en passant par l'hôtel de ville délibératif de la Nouvelle-Angleterre virtuelle, qui croîtront ou s'évaporeront dynamiquement selon les préférences et expériences des utilisateurs.

La nature de l'univers des mondes virtuels a d'importantes implications pour le rôle (et l'efficacité) ou pour les régulateurs du monde réel. Au moins, un marché concurrentiel de mondes virtuels n'implique pas que les régulateurs du monde réel mettent en place des dispositions antitrust. Il existe toutefois un autre aspect du paysage de mondes virtuels, qui apparemment est moins dynamique que ce que j'avais prévu,

\section{Second Life et le " piège de Microsoft "}

Le contenu reste la principale différence entre les mondes virtuels, bien que les fournisseurs 
aient peu d'influence directe sur celui-ci. Leur contrôle indirect, en revanche, est important, car plus il y d'utilisateurs actifs, plus il y a de contenu créé. Nous le savions depuis longtemps : les mondes virtuels sont semblables aux autres industries en réseau. Plus le nombre d'entrants est important, plus l'attraction pour le réseau est forte. Mais les mondes virtuels sont des réseaux avec une torsion. Ils permettent aux utilisateurs de s'appuyer sur l'infrastructure qu'ils fournissent. Par conséquent, les mondes virtuels ne sont pas seulement des plates-formes de réseaux, mais pour l'activité des tiers (c'est-à-dire les utilisateurs), et en tant que plates-formes, ils créent des systèmes rigides de protection et de verrouillage. De manière un peu provocatrice, Second Life en tant que plate-forme est en passe d'être au marché des mondes virtuels ce que Microsoft est pour les systèmes d'exploitation et les applications de bureautique, ou Google pour les moteurs de recherche sur Internet.

Les conséquences pour toutes les parties en présence sont graves. En concurrence avec les fournisseurs de Second Life, les nouveaux entrants, en particulier, auront du mal à accroître leur base d'utilisateurs. L'usager se sentira enfermé, n'ayant plus de choix. Les décideurs politiques du monde réel devraient prendre en compte cette nécessité de réglementer les fournisseurs de mondes virtuels, en particulier Linden Lab, afin d'assurer un minimum de la concurrence (et donc de choix pour l'utilisateur), plutôt que de focaliser leurs attentions sur les modalités de réglementations des biens virtuels contre Linden Lab. C'est donc comme si les politiques se réveillaient et réalisaient que cette année était celle de Microsoft.
La façon la plus évidente pour éviter le « piège Microsoft » est de réduire la plate-forme de verrouillage, par exemple par la mise en œuvre de mécanismes techniques solides qui faciliteraient la migration d'un monde virtuel à un autre, en intégrant le transfert de biens virtuels. Cette interopérabilité, première étape nécessaire, n'est en soi pas suffisante. Alors que les législateurs du monde réels essaient de déterminer comment dépasser le problème du verrouillage de plateforme, - leur bilan n'est pas impressionnant, comme l'illustrent les exemples de Microsoft et de Google -, devons-nous attendre une innovation de rupture (qu'elle soit technique ou organisationnelle, comme l'open source ou la peer production $^{9}$ ) qui remplace un monde virtuel dominant par un autre, dans l'espoir qu'à long terme, les choses fonctionnent? Peut-être.

Il existe toutefois une autre solution afin d'éviter le piège Microsoft, mais qui n'est pas parfaitement évidente. Il pourrait être dans l'intérêt de Linden Lab de réduire délibérément le contrôle de Second Life sur leur base d'utilisateurs - pour deux raisons importantes. Tout d'abord, dynamiser le paysage concurrentiel de mondes virtuels réduit la pression qui pèse sur Linden Lab : être réglementé en tant qu'unique fournisseur de plate-forme en situation de monopole. C'est une dynamique pro-active que Microsoft a malencontreusement évité (si l'on ne tient pas compte des investissements limités dans Apple). Deuxièmement, il sera plus difficile pour les politiques de réguler une multiplicité de fournisseurs de mondes virtuels qu'un seul fournisseur en position dominante. La concurrence des mondes virtuels réduit considérablement les risques pour Linden Lab d'être la cible unique des mesures réglemen- 
taires, dont la portée doit être bien plus grande que l'établissement d'un marché concurrentiel dans le paysage des mondes virtuels.

La clé réside dans la multiplication des fournisseurs de mondes virtuels sur un mode concurrentiel, tout en assurant, grâce à l'interopérabilité des technologies, que les utilisateurs puissent facilement migrer et transférer leurs biens virtuels d'un monde à l'autre. Certains facteurs suggèrent que Linden Lab a l'intention d'adopter une telle stratégie. Ils ont ouvert le code source du logiciel client (qui fonctionne sur les ordinateurs des utilisateurs) et ont renforcé l'interopérabilité du code basique de leurs serveurs. Ils ont laissé entendre qu'ils vont transformer le code basique et la licence du serveur open-source pour que d'autres acteurs puissent devenir les fournisseurs de mondes virtuels, tout en gardant des avantages compétitifs, en se gardant le droit d'ajuster le serveur et le code de visualisation (tant que l'interopérabilité est maintenue). Et ils ont rejoint (comme je l'ai mentionné) un groupe de sociétés, notamment IBM, développant des normes d'interopérabilité pour les mondes virtuels.

C'est certainement une stratégie risquée. Cela implique de renoncer au contrôle quotidien de son monde virtuel (ou des parties de celui-ci) alors que certains utilisateurs pourraient continuer à considérer Linden Lab comme leur fournisseur. Cela implique l'adoption d'un modèle de revenus à adopter excluant les frais d'utilisation directe, et qui tendrait sur le modèle de la licence d'exploitation ou autres modalités de paiement pour les fournisseurs de Linden Lab. Il faudra être d'autant plus vigilant lorsqu'ils intégreront, dans le code logiciel de base, la sécurité, la confidentialité et la stabilité (actuellement, ces qualités peuvent être maintenues pour le prestataire). Pourtant, compte tenu de la position dominante de Second Life, cela pourrait être pour Linden Lab le meilleur pari économique. Mais cela requiert de la vigueur. Certains prédisent déjà que Linden Lab serait sur la réserve. Récemment, le directeur technique Cory Ondrejka a quitté l'entreprise. Son départ, décidé par le fondateur de Linden Lab, Philip Rosedale, est un fait emblématique de la situation, Ondrejka étant le défenseur principal de l'interopérabilité et de l'open-source. Le temps nous le dira.

\section{Conclusion}

Dans ce court essai, j'ai défini le paysage actuel des mondes virtuels, pourquoi et comment la dynamique concurrentielle entre les fournisseurs de mondes virtuels a des conséquences pour les régulateurs du monde réel ainsi que pour les 20 millions d'utilisateurs. J'ai suggéré que le paysage émergent des mondes virtuels est non seulement un terrain intéressant pour les théories de l'économie politique internationale, mais aussi pour le cadre réglementaire de la concurrence, concernant notamment les plates-formes.

Artisans de la réussite à long terme, les fournisseurs de mondes virtuels (en particulier les dominants) pourraient vouloir abandonner leur contrôle (au moins dans une certaine mesure), tandis que les décideurs politiques se retrouveront face à un choix cornélien qui déterminera la plate-forme qui obtiendra le monopole, mais à des niveaux encore plus élevés, non plus seulement d'un point de vue réglementaire, mais avant tout d'une certaine complexité conceptuelle. 
$\mathrm{N} \cdot \mathrm{O} \cdot \mathrm{T} \cdot \mathrm{E} \cdot \mathrm{S}$

1. Voir par exemple : Lastowka, Gregory \& Hunter, Dan, « The Laws of the Virtual Worlds », California Law Review, ${ }^{\circ} 1,2004$;

Lastowka, Gregory \& Hunter, Dan, « Virtual Crimes », New York Law School Law Review, n² 293, 2004 ;

Balkin, Jack, « Virtual Liberty: Freedom to Design and Freedom to Play in Virtual Worlds », Virginia Law Review $\mathrm{n}^{\circ}$ 2043, 2004 ;

Glushko, Bobby, Note: « Tales of the (Virtual) City: Governing Property Disputes In Virtual Worlds», Berkeley Tech. L.J. $\mathrm{n}^{\circ}$ 507, 2007.

2. Ondrejka, Cory, « Escaping the Gilded Cage: User Created Content and Building the Metaverse », New York Law School Law Review, n81, 2004.

3. Ondrejka, op. cit.

4. Mayer-Schönberger, Viktor \& Crowley, John, « Napster's Second Life? The Regulatory Challenges of Virtual Worlds ", 100 Northwestern law Review 1775, 2006.

5. Naone, Erica, « Moving Freely between Virtual Worlds », Technology Review, October 29, 2007.

6 . Voir essentiellement Lazer, David, « Regulatory Interdependence and International Governance », 8 Journal of European Public Policy 474 (2001).

7. Voir par exemple Roe, Mark, «Delaware's Competition », 117 Harvard Law Review 588, 2003.

8. Tiebout, Charles M., «A Pure Theory of Local Expenditures », 64 Journal of Political Economics 416, 1956.

9. NDT : littéralement la «production par pairs». La peer production est un concept de production économique élaboré par Yochai Benkler désignant la production de biens de grande envergure, par l'usage collaboratif d'un nombre important de personnes, coordonnées par un outil (Internet), sans hiérarchie ni aide financière. Benkler, Yochai, The Wealth of Networks: How Social Production Transforms Markets and Freedom, Yale University Press, 2006. 
$R \cdot E ́ \cdot S \cdot U \cdot M \cdot E ́$

Les mondes virtuels offrent une perspective fascinante sur les dynamiques de régulation entre juridiction en compétition. D’une manière plus générale se pose la question des effets de ces jeux sur les régulateurs du monde réel et les dizaines de millions d'utilisateurs. Cet article postule que les fournisseurs de mondes virtuels (en particuliers les dominants), devrait céder une part du contrôle (dans une certaine mesure). Ainsi les décideurs publics du monde réel se retrouveront en partie dans le choix performatifs des plates-formes monopolistiques, et ce à un niveau supérieur de régulation, mais aussi dans une certaine complexité conceptuelle.

\section{After the Constitutional Moment : Regulating Virtual}

Worlds 2.0

Virtual worlds offer a fascinating perspective on regulatory dynamics between competing jurisdictions. More importantly, how these dynamics play out has vast consequences for real world regulators and tens of millions of users. I suggest that virtual world providers (especially dominant ones) may want to cede control (at least to an extent), while real world policy makers will find themselves in a replay of injecting choice into platform monopolies at higher levels of not just regulatory, but also conceptual complexity. 